\title{
Nano Reinforcements in Surface Coatings and Composite Interphases
}

\author{
Shang-Lin Gao and Edith Mäder \\ Leibniz-Institut für Polymerforschung Dresden e.V.,
}

Germany

\section{Introduction}

Nano reinforcements have aroused considerable attentions from both material science and engineering application points of view because of their extraordinary mechanical properties and electronic structures. The use of nanoclays, carbon nanotube networks in surface coatings and composite interphases is leading to rapidly growing unique areas of nanotechnology, especially in integrating mechanical and electrical functionality on the nanoscale. A more comprehensive understanding of the role of nano reinforcements on material surface defect repairing and the realization of functional composite interphase is a significant research focus.

Most solid materials have surface defects. In brittle materials such as glass and ceramics, a fundamental mechanism of failure is the spreading of surface defects. Surface defects of brittle materials cause actual tensile strength much lower than the ultimate theoretical strength. The surface defects normally on the nanoscale providing extra stress at the tip of the cracks can lead to stress-corrosion cracking at low stress level. Generally, polymer coatings are always applied to various fibres to protect them from mechanical damage during handling. Such coatings also act as a diffusion barrier to moisture reaching the fibre surface from the surrounding environment. Healing nanoscale surface flaws and enhance materials' lifetime by coating, therefore, are important for many traditional materials for wide use in aggressive environments. The mechanical 'healing' effect was viewed as a disappearance of the severe surface flaws because of an increase of the crack tip radius, the flaw filled by coatings being either elliptical than sharp. Reinforcement with nanomaterials in coatings is a topic of significant current interest. The molecular dynamics simulations show that the stress concentration at the notch tip is significantly reduced due to the presence of the nanoparticles (Tyagi et al., 2004). These results point to a simple means of fabricating systems that can self-heal, where nanoparticles dispersed in a polymer matrix can migrate to a crack generated at the interface between the polymer and a glassy layer (Gupta et al., 2006). Fundamentally, optimised mechanical structure and nanostructured surface are motivated by the grace and efficiency of natural materials, in a biomimetics approach. It is evolved in these materials, as shown in Fig. 1 (left), that multiscale structures (i.e. bones) and skin coverings with flexible multilayer of overlapping tough scales (vertebrates, i.e. fishes) provide a protective layer against physical/chemical attack. The nanometer size of mineral particles, composed of insoluble protein keratin and minerals, ensures optimum strength and maximum tolerance of flaws (Gao et al., 2003). As the 
structural size shrinks to the nanometer scale, there is a transition of fracture mechanism from the classical Griffith linear elastic fracture mechanics (LEFM) to one of homogeneous failure with no stress concentration at the crack tip and the structure becomes insensitive to pre-existing flaw (Gao \& Ji, 2003). The surface free energy becomes more dominant and the material strength is limited by theoretical strength of solid. The surface defect-free and high purity carbon nanotubes have exceptional high Young's modulus and tensile strength. However, an effective utilization of their mechanical properties in composites is a long standing problem. To date, the highest strength and Young's modulus reported in the literature are relatively disappointing: 1.8 3.2 GPa and $\sim 40 \mathrm{GPa}$, respectively, for aligned nanotube composite bundles with very high volume loading of nanotubes (60 wt\%), which are a factor of ten below those of the component individual nanotubes (Vigolo et al., 2000, 2002). Our recent work applied a 'surface defects healed by super-materials' approach to glass fibres since glass fibres are most widely used traditional reinforcements in composites globally (Gao et al., 2007). Using nanotubes in coatings to heal common continuous glass fibre, strengthening as much as $60 \%$ is achieved because surface coatings can efficiently protect the fibre surface against alkali/acid/moisture and thus improve fibre's mechanical properties. The mechanical properties of the healed glass fibres are equal and even higher than the corresponding values of aforementioned high volume concentration bundled nanotubes.
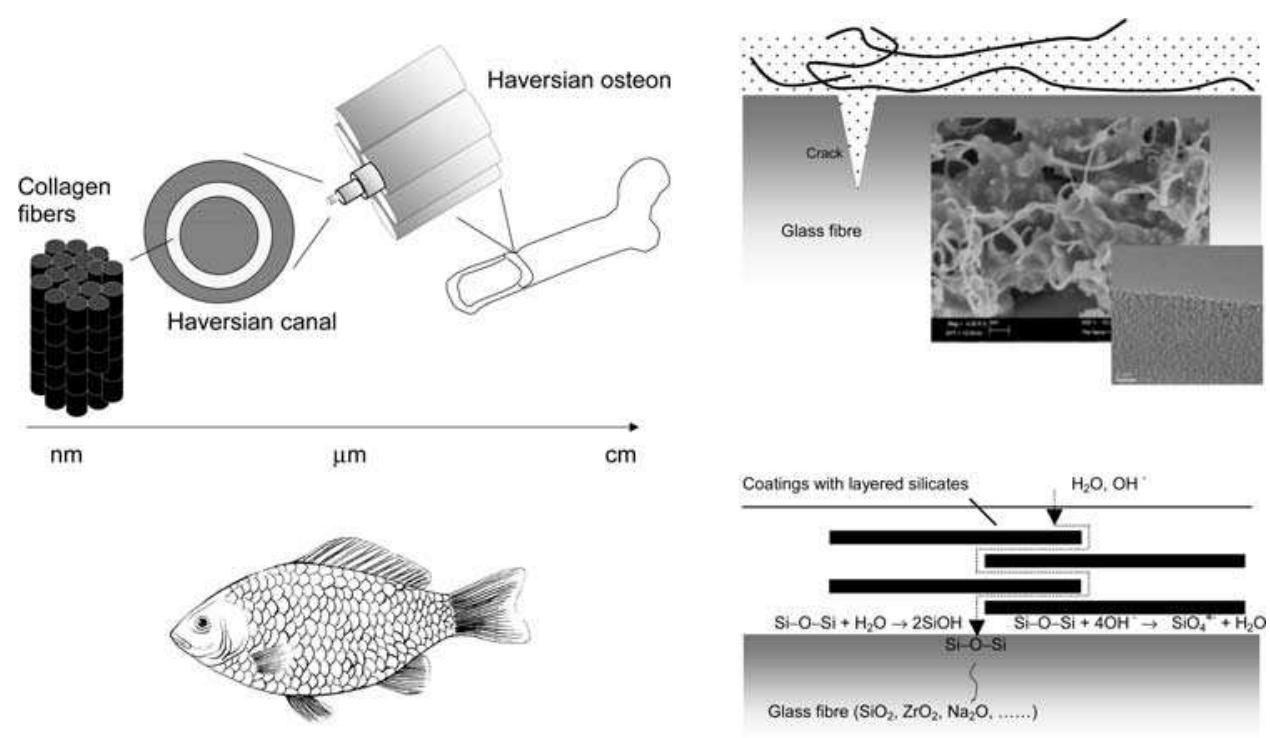

Fig. 1. Natural strategy of mechanical reinforcement and environmental resistance by multiscale fibres and overlapping tough scales (left). Applications of nanostructed coatings with nanotube/layered silicate polymer network on glass fibre surface to enhance healing flaw effect and corrosion resistance (right). The inserts show polymer/multi-walled carbon nanotubes (MWCNT) network by SEM and individual surface functionalized nanotube structure by TEM. 
Another fundamental feature of glass fibre is electrical insulating. The development of novel glass fibre reinforced plastics (GFRPs) with electrical conductivity has opened up new opportunities in which unique functionality can be added to existing material systems for a broad range of applications, including electrostatic dissipation, electric field shielding, damage detecting, etc. As pilot approach, the electrical conductivity of GFRPs has been achieved by either adding conductive particles, such as carbon blacks and carbon nanotubes (CNTs) in polymer matrix, or composite surface treated with antistatic or metallic coatings (Thostenson et al., 2006, 2008, Böger et al., 2008). As outstanding multifunctional sensing materials, recently, CNTs have stimulated the development of various conductive nanotube/polymer composites with sensitive features to piezoresistivity, temperature and moisture (Baughman et al., 2002; Bekyarova et al., 2007; Veedu et al., 2006; Dzenis, 2008). The piezoresistivity of carbon nanotubes has been known for several years, i.e., nanotube electrical resistance changing by mechanical stress (Tombler et al., 2000). The GFRPs with carbon blacks and MWCNTs in polymer matrix with health monitoring functionality have recently been successfully developed to monitor damage initiation and evolution. Thostenson and Chou processed GFRPs with embedded CNTs in epoxy matrix to evaluate the onset and evaluation of damage (Thostenson et al., 2006, 2008). One outstanding challenge is to fabricate in-situ sensing composite materials at micrometer scale, especially at interphase of composites (Gao et al., 2010; Zhang et al., 2010), being capable of detecting stress/strain, temperature and humidity with high sensitivity.

Herein, we describe a method that introduces electrical conductivity to glass fibre surface by depositing MWCNT networks, and in turn, specifically forming an interconnected MWCNTs-rich interphase within glass fibre reinforced epoxy composites, showing the potential to realize multi-scale fibre reinforced composites with multifunctional properties. Aimed at multifunctional applications, we performed in-situ DC electrical resistance measurements of the single MWCNTs-glass fibre and 'unidirectional' MWCNTs-glass fibre reinforced epoxy composites at different temperatures, relative humidities ( $\mathrm{RH}$ ), fibre orientations and tensile strains underwent uniaxial tensile or cyclic loading, respectively. The surface morphology of the fibres, interphase properties and the glass transition temperature of composites were studied by ultra high resolution field emission scanning electron microscopy (FE-SEM) and atomic force microscopy (AFM), electric force microscopy (EFM), nanoindentation and differential scanning calorimetry (DSC), respectively. Our approach will help to validate and improve the technique of in-situ monitoring/sensing in advanced nanocomposites, implying highly sensitive to the fracture of the load-carrying fibres and the development of cracks in the fibre/polymer matrix interphases, where the microscale damage is usually initiated.

\section{Experimental}

\subsection{Glass fibre coated by polymers and MWCNTs or clay}

The control alkali-resistant glass (ARG) fibres utilized in this work were made at our institute with diameters of $17 \mu \mathrm{m}$. During the continuous spinning process, the ARG fibres were in-situ sized by an alkali-resistant sizing consisting of silane coupling agent, $\gamma$ aminopropyl-triethoxysilane, in conjunction with film formers and nanoparticles in the aqueous sizing, namely S1. The $0.2 \mathrm{wt} \%$ surface functionalized MWCNTs (IFW, Germany) are dispersed in the epoxy film former based sizing. We applied surface coatings to the control ARG using either two kinds of styrene-butadiene copolymers with different $\mathrm{T}_{\mathrm{g}}$ 
values (C1) or a commercial self-crosslinking styrene-butadiene copolymers (C2). Similarly, E-glass fibres with diameters of $20 \sim 23 \mu \mathrm{m}$ were also coated with less than $0.5 \mathrm{wt} \%$ nanotubes in the coatings. The organo-clay particles (Nanofil 15, Süd-Chemie AG, Moosburg, Germany) in maleic anhydride grafted polypropylene with a size of about 60 to $130 \mathrm{~nm}$ are dispersed in the obtained solution. A quaternary ammonium surfactant and a non-ionic surface active agent were added to the dispersion for homogeneous distribution of the constituents with or without $1 \mathrm{wt} \%$ nanoclay. This method benefits from its ambient temperature treatment and environmentally friendly deposition, in addition to chemical versatility. The total weight gain due to the coatings is $5.3 \mathrm{wt} \%$ measured by pyrolysis $(600$ ${ }^{\circ} \mathrm{C}, 60 \mathrm{~min}$ ) of the coated fibres. We extracted the fibres in selected highly concentrated aqueous alkaline solution ( $5 \mathrm{wt} \% \mathrm{NaOH}, \mathrm{pH}$ of 14$)$ at $20{ }^{\circ} \mathrm{C}$ for seven days, which is the most aggressive and corrosive condition to the fibre surface.

\subsection{Glass fibre coated by MWCNTs and glass fibre/epoxy composites}

Briefly, various aqueous dispersions with the $\mathrm{pH}$ value of $5 \sim 6$ and $0.5 \mathrm{wt} \%$ carboxyl functionalized MWCNTs (NC-3101, Nanocyl S.A., Belgium) were utilized for ensuring more homogenous distribution of CNTs on glass fibre surfaces. The dispersion aids are non-ionic, cationic or anionic surfactants, namely Igepal CO970 (shown schematically in Fig. 2(a)), Arquad S-50, and sodium dodecyl sulfate (SDS), respectively. The glass fibres were dipped into MWCNTs dispersion for $15 \mathrm{~min}$ and dried in a vacuum oven for $8 \mathrm{~h}$. A commercial DGEBA-based epoxy with amine hardener (EPR L20, EPH 960, Hexion Speciality Chemicals $\mathrm{GmbH}$, Germany) in a weight ratio of 100:34 was used as matrix and the single glass fibre and unidirectional MWCNTs-glass fibre reinforced epoxy composites over a very wide range of glass fibre volume fractions from $4 \times 10^{-3} \%$ to $50 \%$ were cured at identical conditions $\left(80{ }^{\circ} \mathrm{C}, 6\right.$ h), where no additional CNTs were added to the matrix. For electrophoretic deposition (EPD) coating, silane coupling agent 3-Glycidyloxypropyltrimethoxysilane (Dynasylan® Glymo, Evonik Degussa Corporation, Germany) was added into CNT dispersion to introduce functional groups onto fibre surface and to improve the interfacial shear strength. The concentration of MWCNTs was as small as $0.05 \mathrm{wt} \%$. We used two parallel copper plates with rectangular-shape as cathode and anode. According to streaming potential results, the dispersed MWCNTs and the hydrolyzed Glymo showed negative charge and migrated towards the positive electrode, the anode was used as deposition electrode, glass fibres were fixed on a thin plastic frame, and then the frame was mounted on the anode, which is schematically shown in Fig. 2(b). EPD experiments were carried out at constant voltages, deposition time of $10 \mathrm{~min}$, and an electrode distance of $8 \mathrm{~mm}$. The coated samples were dried at $40^{\circ} \mathrm{C}$ in a vacuum oven for $8 \mathrm{~h}$. Due to their very small size and well-dispersed state, the carbon nanotubes were able to penetrate into the spaces between the fibres and coagulate on the whole surface of fibre.

\subsection{Characterisation}

The MWCNTs-glass fibre surface and composite interphase properties were studied using ultra high resolution field emission scanning electron microscopy (FE-SEM Ultra 55, Carl Zeiss SMT AG, Germany) and AFM (a Digital Instruments D3100, USA). AFM modes of tapping, LiftMode electric force microscopy (EFM) and nanoindentation were performed to obtain topography morphological, electric force images and nanomechanical stiffness, respectively. To assure good topography imaging resolution and nanometer scale indents, 


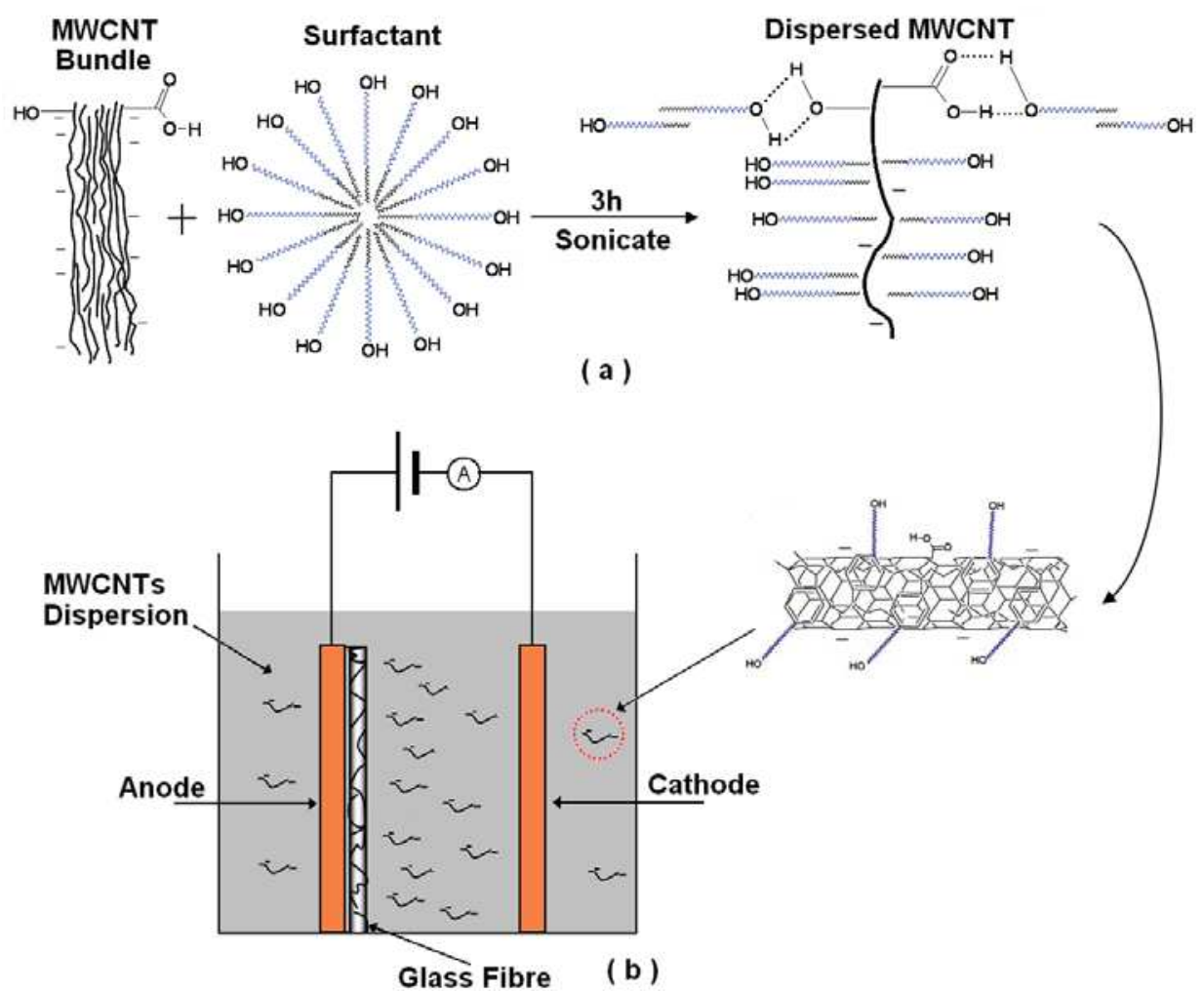

Fig. 2. Schematic illustrations of (a) MWCNTs dispersion process in water with surfactant and (b) deposition of MWCNTs onto insulative glass fibre surface by the electrophoretic deposition cell.

the ultra-sharp cantilever (NSC15-F/5, MikroMasch, Estonia) has a radius of $\sim 10 \mathrm{~nm}$, a normal spring constant of $40.9 \mathrm{~N} / \mathrm{m}$ and modulus of $160 \mathrm{GPa}$. An electrical field can be induced by applying a voltage of $12 \mathrm{~V}$ between an ultra sharp conductive AFM tip (NSC14/W2C/15, MikroMasch, Estonia) and the nanotube rich interphase, where the tip interacts with the interphase through long-range Coulomb forces at a constant distance of $50 \mathrm{~nm}$. We detected the phase shifts to create EFM images, arising from the interactions changing the oscillation phase of the AFM cantilever, where attractive forces make the cantilever effectively "softer" reducing the cantilever resonant frequency, and conversely repulsive forces make the cantilever effectively "stiffer" increasing the resonant frequency. To ensure a low surface roughness across the interphase, the specimens were polished perpendicularly to the fibre axis with a $\mathrm{SiO}_{2} / \mathrm{Al}_{2} \mathrm{O}_{3}$ suspension down to an average grain size of $60 \mathrm{~nm}$. In addition, the $\mathrm{T}_{\mathrm{g}}$ was measured for the composite sample with fibre volume fraction of $40 \%$ by the modulated DSC (Q2000 MDSC, TA Instruments, USA) at the rate of temperature change of $3 \mathrm{~K} / \mathrm{min}$.

The technique applied to operate and measure the electrical property of a single MWCNTsglass fibre has been realized, using one fibre bridging two $\mathrm{Cu}$ electrodes on epoxy substrate with small gap distances of $0.3,1.0$, and $2.2 \mathrm{~mm}$, respectively. Without using conductive 
silver paste, the fibre was assembled carefully along a narrow channel ditch on the relatively soft $\mathrm{Cu}$ electrodes, which was prefabricated by surface indentation using the same kind of glass fibre. In this configuration a large amount of MWCNTs on the fibre surface was forced to attach to the electrodes under compression. Besides, the surface forces, van der Waals, and capillary forces are enough to establish a sufficiently intimate electrical and mechanical contact between the nanotubes and the electrodes [45]. Approximately ten fibre specimens for each condition were measured. Two-probe setup with a Keithley 2000 multimeter and a DC power supply (ELV PS 7020) was used to obtain the I-V curves. Four-point conductivity measurements were carried out to monitor the DC electrical resistance using a Keithley 2000 multimeter and two-point conductivity was also carried out with LCR-digital multimeter (VC-4095) for the resistance value higher than $100 \mathrm{M} \Omega$ of single MWCNTs-glass fibre. We further performed in-situ electrical resistance measurements of the single MWCNTs-glass fibre at different tensile strains, temperatures, relative humidities $(\mathrm{RH})$. The tensile strength of single fibre was measured using the Favigraph semiautomatic fibre tensile tester (Textechno, Germany) equipped with a $1 \mathrm{~N}$ load cell, according to DIN EN ISO 5079 and DIN 53835-2, respectively. To investigate the piezoresistive effect, the electrical resistance was recorded as the single glass fibre underwent uniaxial tensile or cyclic loading. The tests have gauge length of $20 \mathrm{~mm}$ and the cross head velocity of $0.2 \mathrm{~mm} / \mathrm{min}$ for both loading and unloading with strain amplitude of $2 \%$. To ensure good electrical contact, gold deposition with thickness of about $60 \mathrm{~nm}$ was sputtered to two ends of single MWCNTsglass fibre except of the middle part of fibre with length of $2 \mathrm{~mm}$ for the measurement. The specimen was in turn clamped between two plates coated with conductive silver paste (Acheson Silver DAG 1415M) serving as electrodes. Simultaneous resistance, strain, and load measurements were integrated with time scale in a customized data acquisition package TestPoint 2.0. In order to further detect composite piezoresistive effect, mechanical tensile/compression strains were performed using a self-made screw-driven tensile stage and simultaneous resistance was recorded at each strain step. The experiments of the resistance changing with the temperature were carried out in a hot-stage (Linkam LTS350 Heating/Freezing, UK) from $-150^{\circ} \mathrm{C}$ to $180^{\circ} \mathrm{C}$ with a heating rate of $1 \mathrm{~K} / \mathrm{min}$ in a nitrogen atmosphere. We prepared unidirectional MWCNTs-glass fibre/epoxy composites over a very wide range of volume fractions from $4 \times 10^{-3} \mathrm{vol} \%$ to $50 \mathrm{vol} \%$, including dog-bone shaped samples $\left(20 \times 1 \times 1.8 \mathrm{~mm}^{3}\right)$ and rectangular-shaped samples $\left(20 \times 7 \times 1.8 \mathrm{~mm}^{3}\right)$ for electrical testing. The surface for electrodes was mechanically polished with 2400 grade silicon carbide grinding paper followed by sputter coating of gold layer with thickness of about $60 \mathrm{~nm}$.

\section{Results and discussion}

\subsection{Nano reinforcements in fibre surface coatings: mechanical properties}

We first investigated the tensile performance of the single fibre with nano reinforcements in surface coatings (Fig. 3). In comparison with unsized samples, we observed a significant improvement of $70 \%$ of tensile strength for nanostructured glass fibre with nanotubes. The fibre strength also increased up to $40 \%$ and $25 \%$ for sized fibre and sized fibre with $1 \mathrm{wt} \%$ loading of organoclay in the sizing, respectively. The fibre fracture behaviour is strongly affected by the variation of sizing properties because the critical flaws which limit the strength of fibres are located at the surface. Additionally, the effects of alkaline attacks on the average fibre strength are also compared in Fig. 3. It is evident that sample of clay 
coatings would not yield a significant strength reduction upon alkali treatment. Therefore, the durability and alkali-resistance are also improved, particularly the fibre with organoclay coatings. Overall, the coated fibres have higher strength values than the control one after alkaline corrosion, reflecting the improved environmental durability for fibres with nanostructured coatings.

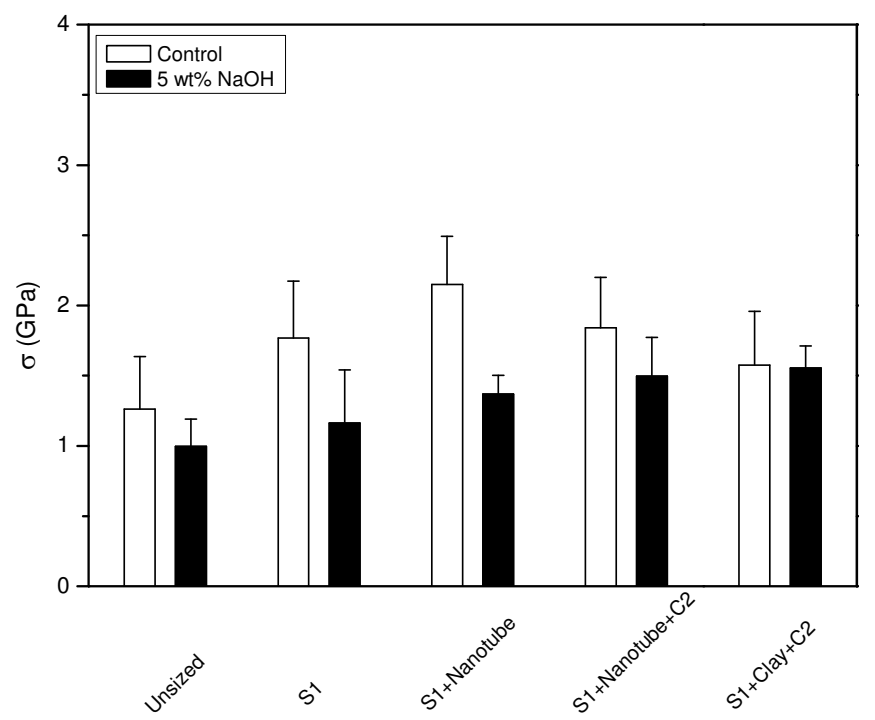

Fig. 3. Effect of the nanostructed coatings with low fraction of nano-reinforcements on the tensile strength of ARG before and after alkaline treatment in $5 \mathrm{wt} \% \mathrm{NaOH}$ aqueous solution for seven days in an ambient environment. Error bars represent standard deviations for the estimate of the mean strength of fifty samples.

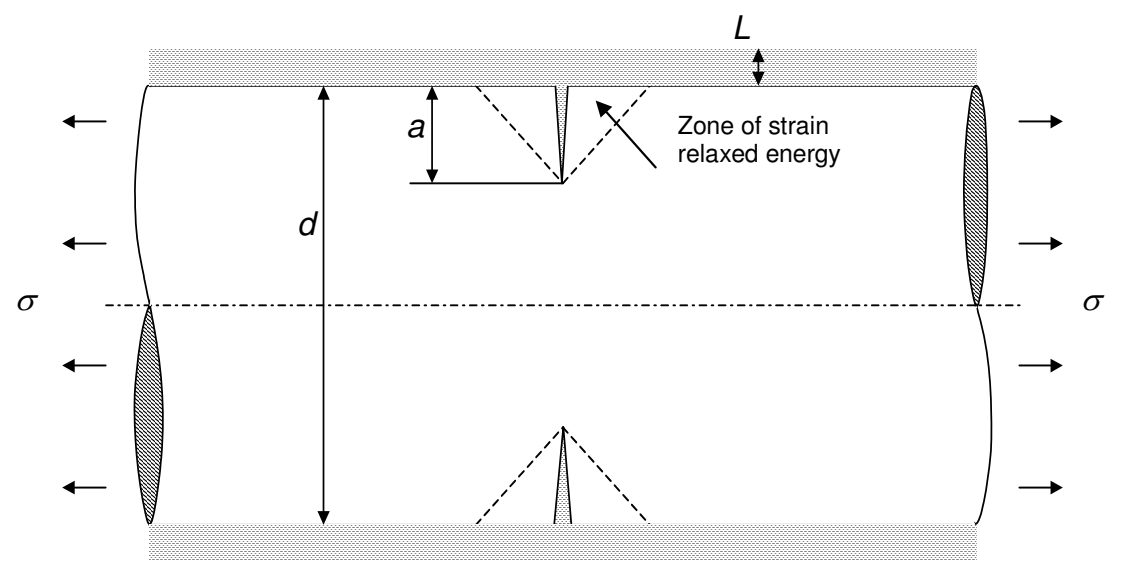

Fig. 4. A sketch of a coated fibre with a surface flaw. The fibre is loaded in tensile stress $\sigma$ and the circumferential surface flaw of length $a$ serves as an initial crack. The fibre diameter and coating thickness are given by $d$ and $L$, respectively, where $a$ and $L$ are much less than $d$. 
Potential mechanisms include the contributions of different factors for the mechanical property improvement by reducing the fibre surface flaw formation and crack growth. Note that the polymer coatings have Young's moduli that are typically several orders of magnitude lower than the glass fibre, and therefore do not bear a significant portion of the mechanical load. Although the polymer coatings do not increase strength, they have the important function of protecting the glass surface from abrasion and chemical damage, which in turn would degrade glass fibre strength. The coating layer with organosilicate plates could prevent moisture/alkali contact and reaction with glass lattice at a crack tip (stress corrosion). The acidic groups of coating molecular interact with or absorb free cations and anions of environment leading to a slow-down of the corrosion process. Secondly, stress-redistribution and crack stopping mechanisms by coatings and nanotube's 'bridging' effect and interface debonding/plastic deformation around crack tip. The mechanical 'healing' effect was viewed as a disappearance of the severe surface flaws because of an increase of the crack tip radius, the flaw filled by coatings being either elliptical than sharp. Thirdly, compressive stress on fibre surface might prevent crack opening/propagation by the shrinkage of polymer due to solidification. Because of the compression closing surface flaw, the strengthening can be increased by increasing the magnitude of the compressive stress.

To simplify the complex phenomena, we developed a simple mechanical model based on Griffith fracture mechanics to roughly estimate the strength of coated fibre. Consider a smoothly coated fibre loaded in tension and having a thin circumferential crack (Fig. 4). When the crack appears, the strain energy is released in a material volume adjacent to the crack. Assume that this volume is comprised by a conical ring whose generating lines are shown by broken lines and heights are proportional to the crack length. The present assumption is arbitrary and significant analogy to the original Griffith strain energy analysis for an elliptical, sharp crack embedded in a flat, brittle sheet. Accordingly, the energy is consumed by formation of new surfaces and deformation of coatings because of an elastic constraint. According to the energy balance, the coated fibre strength, $\sigma_{f}$, can be expressed as

$$
\sigma_{f}>\bar{\sigma}_{f}=\sqrt{\frac{2 \gamma E_{f}}{\left(\beta a^{*}-\frac{L(1+L / d) E_{c}}{E_{f}}\right)}}
$$

where $\gamma$ is fracture surface energy and $\beta\left(=1-2 a^{*} / 3 d\right)$ is a constant coefficient of proportionality which is very close to one since an apparent crack length $a^{*}$ is much less than fibre diameter $d$. We used the apparent crack length $a^{*}$ instead of $a$ to take into account geometrical influences to surface defect arising from either coatings filling of crack tip or surface roughness. $E_{f}$ and $E_{c}$ are Young's modulus of fibre and coatings, respectively. Notably, the critical tensile stress of fibre with a surface flaw, $\bar{\sigma}_{f}$, is significantly affected by the coating modulus and thickness. Generally speaking, the thicker the coating layer and larger the stiffness of coatings the higher is the tensile strength of the fibre. On the other hand, the larger the size of defect and higher the stiffness of fibre, for effective repairing, the thicker and stiffer coatings are required. 


\subsection{Nano reinforcements on fibre surfaces: Electrical properties}

Next, we present the results of the electrical resistance measurement of single glass fibre coated with anionic dispersant individualized MWCNTs (Fig. 5). The measured DC resistances $R$ of the single MWCNTs-glass fibre are in the range of 104 up to $107 \Omega$. It in general increases with increasing electrode-electrode distance, $L$. Accordingly, the calculated specific conductivity $\sigma_{g l a s s}=4 L / \pi R d^{2}$, for our MWCNTs-glass fibre with diameter of $d$, is typically in the range of 0.1 up to $30 \mathrm{~S} \mathrm{~m}^{-1}$ and the fibre surface resistance values are in the range of $10^{3}$ to $10^{7} \Omega / \mathrm{sq}$. The data presented here could be explained most readily if it is assumed that there is a nanotube layer with a thickness, $t$, on fibre surface having electrical conductivity, $\sigma_{c n t}$. The specific conductivity of MWCNTs-glass fibre, $\sigma_{g l a s s}$, parallel to the fibre axis is therefore given by:

$$
\sigma_{\text {glass }}=4\left(\frac{t}{d}+\frac{t^{2}}{d^{2}}\right) \sigma_{c n t} \approx \frac{4 t}{d} \sigma_{c n t}
$$

Taking a rough estimate, an average glass fibre diameter $d \quad 17 \mu \mathrm{m}$ and $t$ is in range of a few tens to a few hundreds of nanometres based on fibre surface roughness data, we see the ultra-thin nanotube networks with $\sigma_{\text {cnt }} 10^{2}$ to $10^{3} \sigma_{\text {glass }} 10$ to $10^{4} \mathrm{~S} \mathrm{~m}^{-1}$. It possesses conductivities approaching to the highest values, typically $10^{4}$ to $10^{5} \mathrm{~S} \mathrm{~m}^{-1}$, of the nanotube only buckypapers achieved with an aggregate of high dense carbon nanotube networks. It suggests that the high conductivity is reached for the carbon nanotube networks with locally isotropic and inhomogeneous distribution on our MWCNTs-glass fibre surface. FE-SEM image (Fig. 5) show that the MWCNTs present in the form of closely packed and highly entangled network structure on the curved fibre surface. The aggregated and individual carbon nanotubes could be clearly seen, which create the conductive pathways.

\subsection{Nano reinforcements in composite interphase: structure, adhesion and functionalization}

The question of whether the nanoscale semiconductive interphase between glass fibre and epoxy matrix could be experimentally observed is interesting. Our work highlights the importance of no-contact LiftMode electric force microscopy (EFM) as highly sensitive analytical tools in characterisation of interphase. To assess the variation in properties across the interphase, we investigated the cross-section of MWCNT-glass/epoxy composites by both EFM and FE-SEM (Fig. 6). The ultra high resolution FE-SEM image shows clearly many particles in the interphase region with size from approximately ten to several tens of nanometers, which are likely resulted from the exposed ends of nanotubes. To further examine and confirm, we examined the interphase by EFM (Fig. 6b,c), which allows the imaging of relatively weak but long range electrostatic interactions arising from the semiconductive interphase while minimizing the influence of topography since the tip has a distance of $50 \mathrm{~nm}$ from sample surface (Fig. 6c). The EFM images were created by the phase shifts, arising from the interactions changing the oscillation phase of the AFM cantilever by applying a voltage between an ultra sharp conductive AFM tip and the interphase. It is clear that the EFM image shows apparent contrast between the fibre, interphase and matrix regions, revealing a difference in the material properties of these three regions. The transition "river-like" layer along the fibre surface is attributable to MWCNTs in the quasi$2 \mathrm{D}$ confined interphase region with irregular shape of thickness ranging from $20 \sim 500 \mathrm{~nm}$ 


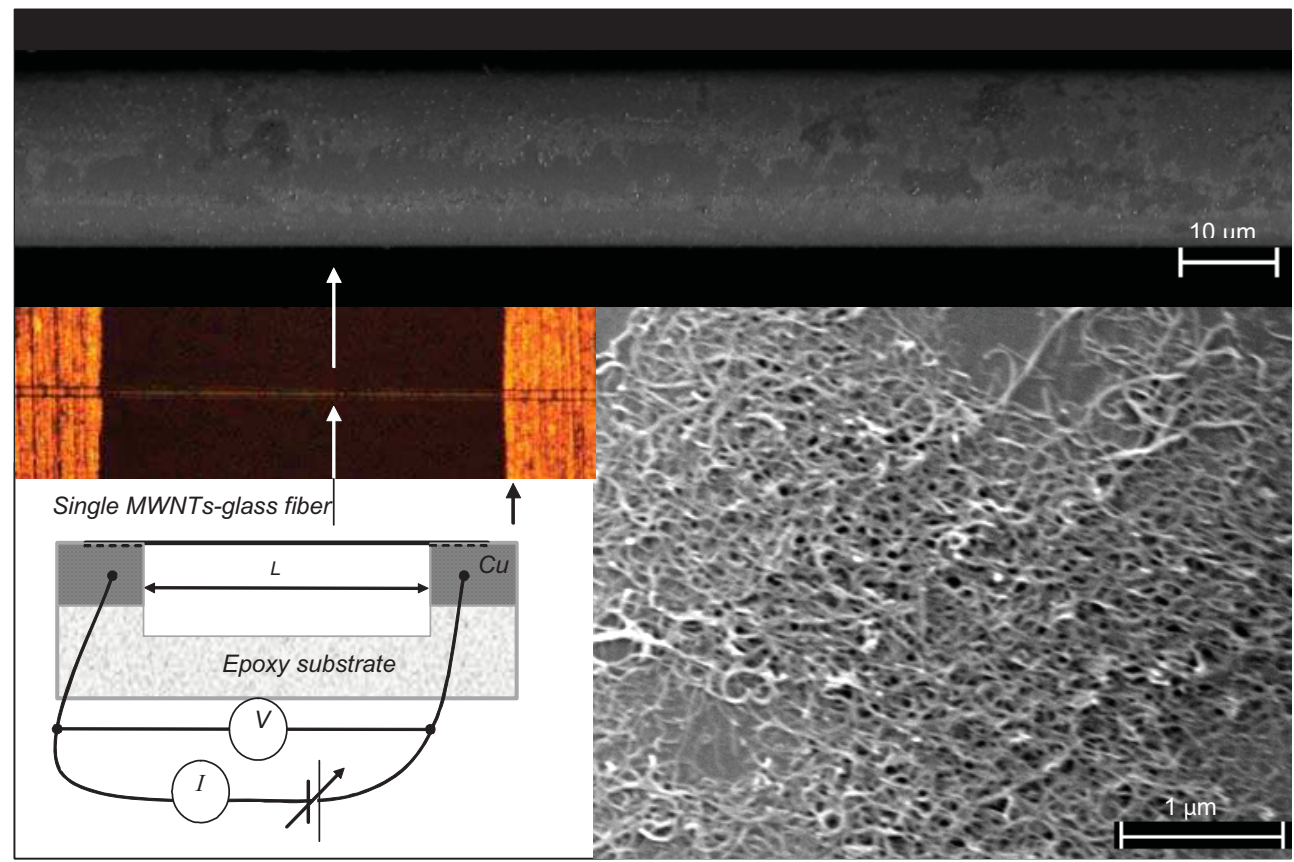

a)

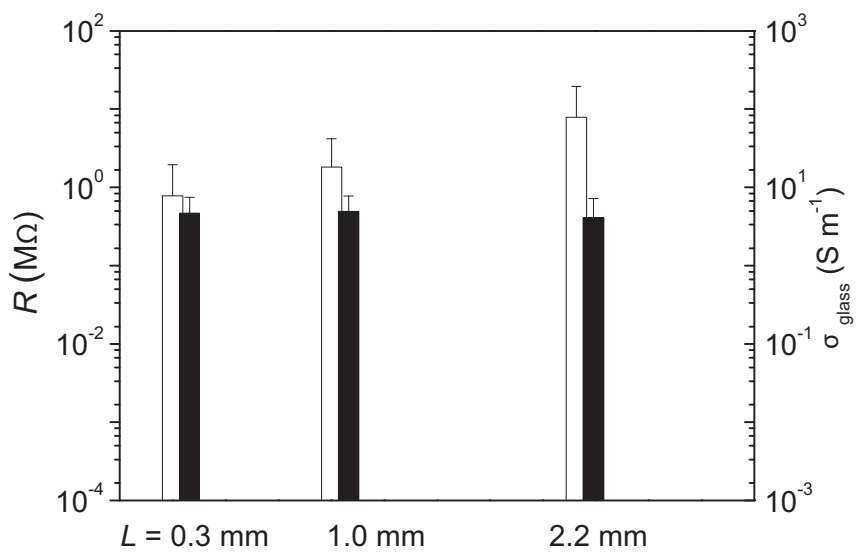

b)

Fig. 5. Electronic transport property of individual single MWCNTs-glass fibre. a) Schematic diagram for the measurements. The upper optical image of the glass fibre between two $\mathrm{Cu}$ electrodes. The low and high-magnification FE-SEM images highlight an inhomogeneous distribution and randomly oriented interpenetrating MWCNT network structure on the glass fibre surface. $\mathrm{b}$ ) The resistance $R$ (white bars) and the specific conductivity $\sigma_{\text {glass }}$ (black bars) versus length $L$ of MWCNTs-glass fibre. Error bars, s.d. 


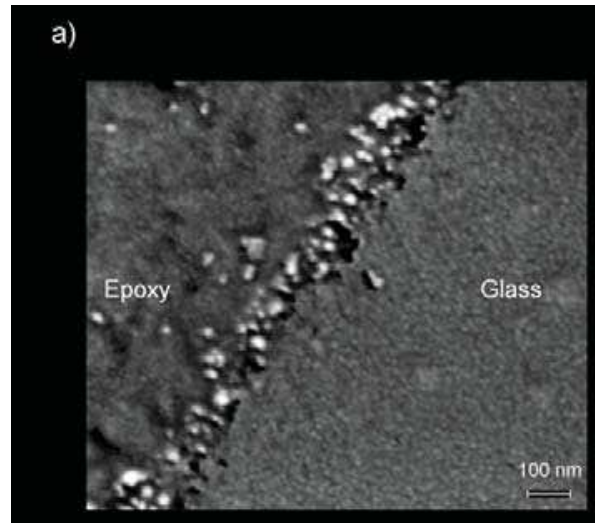

b)

c)

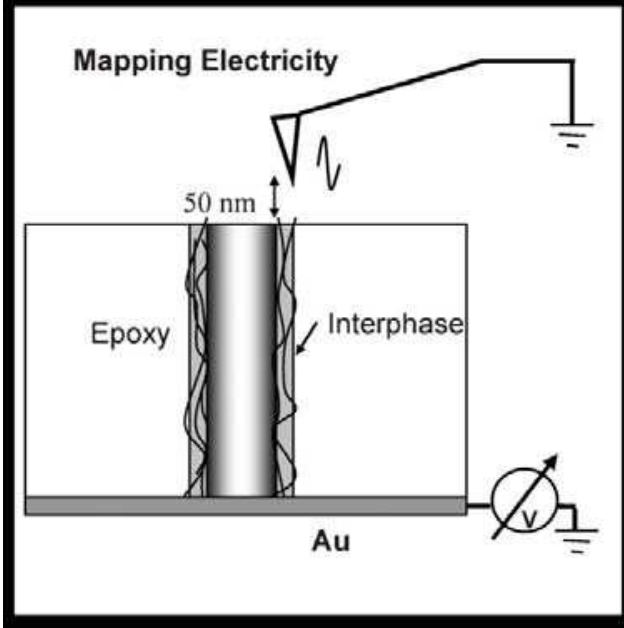

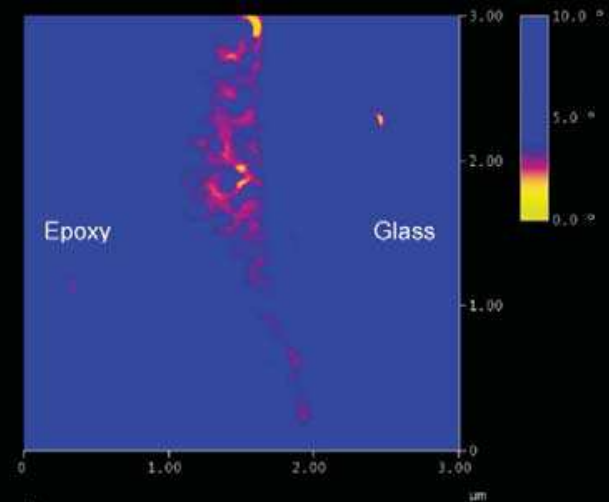

d)

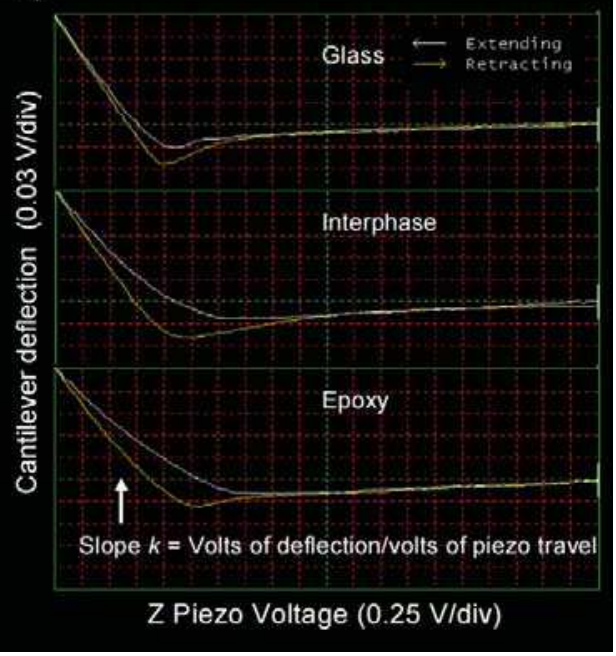

Fig. 6. a) FE-SEM and b) EFM images of MWCNT-rich interphase in MWCNT-glass/epoxy composite; c) schematic diagram for electrical mapping cross section of composites with MWCNT-rich interphase by EFM; d) typical nanoindentation force curves on fibre, interphase, and epoxy represent the cantilever deflection signal for one complete extension/retraction indentation cycle of the piezo. The initial slope $k$ of the retracting curve represents the cantilever deflection signal versus voltage applied to the piezo (i.e., indentation displacement in the vertical direction). A softer material would result in less deflection of the cantilever under a given indentation displacement, which provides qualitative information about the elasticity of specimen surface. The slope of indentation on the interphase showing higher value than that of indentation on the epoxy matrix actually demonstrates the higher stiffness of interphase. 
and above, which is further confirmed by higher contact stiffness at this region in comparison with that of bulk matrix by nanoindentation (Fig. 6d). The higher stiffness of the interphase determined from the slope of curve in Figure $6 \mathrm{~d}$ is related to contribution from the intrinsic high stiffness of nanotubes. The combination of high conductivity and high stiffness within interphase is not found for other reported CNT/polymer composites.

The next work focuses on detecting local thermal properties at sub-100 nm resolution. The nano-TA is a newly available local thermal analysis technique which combines the high spatial resolution imaging capabilities of atomic force microscopy with the ability to obtain understanding of the thermal behaviour of materials, such as melting or glass transitions with a spatial resolution of sub- $100 \mathrm{~nm}$. In this test, the probe in contact with the polymer is deflected, as its temperature is ramped up, and the cantilever sensor displacement in the Zaxis is measured. As the material under the probe is heated it expands, deflecting the probe upward. The surface layer of the polymer then softens leading to plastic deformation under mechanical pressure of the probe. The preliminary results shown in Fig. 7 suggest an increase of the local glass transition temperature as well as a reducing remarkably downward deflection of the probe tip (much smaller penetration holes) in interphase regin, especially in distance less than $500 \mathrm{~nm}$ from the fibre surface. This increasing $\mathrm{T}_{\mathrm{g}}$ assigned to the interphase is probably a consequence of a greater enrichment of amines adsorbed by both nanotube and glass fibre surfaces, resulting higher crosslink density in the interphase region.

The fragmentation test has been used for assessing interfacial shear strength, where the tensile load in the specimen is transferred to the fibre by shear stresses in the matrix through the interphase. The fibre keeps breaking until the fragments become too short to build up sufficiently high tensile load to cause further fragmentation with increasing specimen strain. As expected, the control fibre without MWCNTs had the highest critical aspect ratio, which corresponded to the lowest interfacial shear strength. In contrast, the interfacial shear strength was enhanced by the MWCNT coating, particularly the sample with Glymo achieved the maximum interfacial shear strength. The reason of enhancement might arise from different nanotube related toughening mechanisms, including glass fibre/nanotube/matrix interfacial debonding, nanotube pull-out, interfacial crack bridging, etc. The micro-mechanical interlocking contributes mainly to the frictional bond after fibre debonding, an effect similar to the clench of gears may exist between CNTs and cross-linked epoxy molecules. The potential chemical reactions between epoxy and the carboxy-functionalized MWCNTs as well as hydrogen bonding contributed to the improvement of the interfacial strength as well. In presence of silane coupling agent, the reactions of epoxide-carboxy, epoxy-amine, and silanolsilanol gave rise to chemically covalent bonds around glass fibre, MWCNT, surfactant, and epoxy.

Furthermore, the morphology of MWCNT coating on the fibre surface influenced interfacial shear strength. In these cases, the irregular MWCNT distribution along the fibre causes nonuniform interphase structures and properties (strength/stiffness). We then proposed three different interphase structures: (1) homogeneous interphase; (2) mid-homogeneous interphase; (3) inhomogeneous interphase. Fig. 8 shows the birefringence patterns under polarized light of single fibre model composites together with the schemes for these proposed interphases and the stress profiles along the fibre when the fragment number reached saturation. Clearly, the stress birefringence of control fibres and DIP-coated fibres suggests that the interphases suffered from extensive shear stresses and the crack tended to expand along the interphase. Through focusing on the fibre break point, the apparent matrix crack failure mode could be observed in the coated fibre samples, which indicated improved 


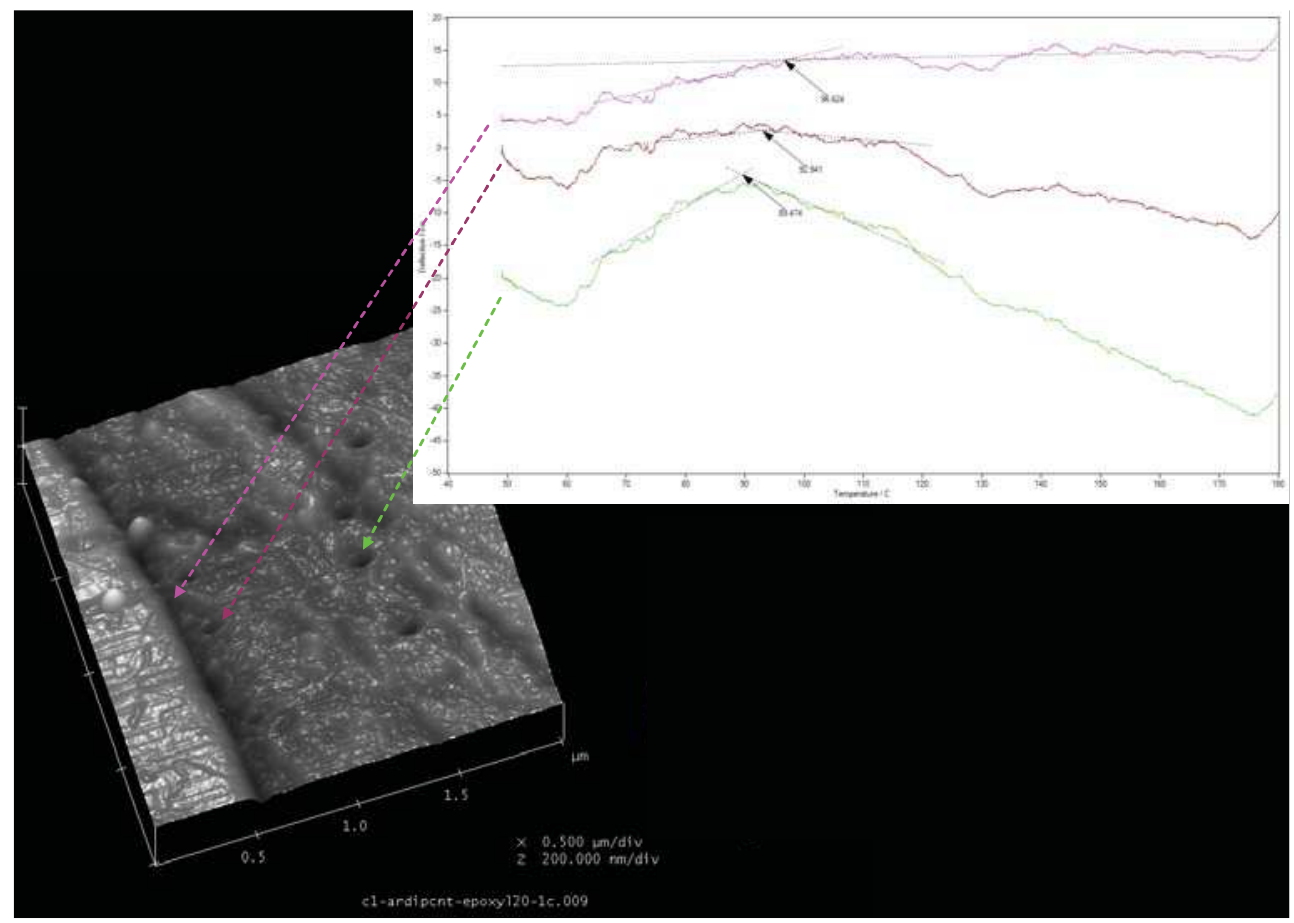

Fig. 7. Nano-TA thermal analysis on a MWCNTs-glass fibre/epoxy resin composite: Sensor height position response versus temperature for different positions from interphase close to glass fibre towards bulk resin. Associated penetration holes shown in AFM topography becoming bigger with increasing distance from the fibre surface.

interfacial strength due to the presence of the MWCNT coating. The control fibre with homogeneous surface possessed the highest value of the Weibull shape parameter $m$, suggesting uniform interfacial adhesion (Zhang et al., 2010). The interphases for fibres treated by EPD method were classified into the mid-homogeneous, since their Weibull shape parameter value is between control fibre and DIP fibre. Due to the differences in thickness of the MWCNT layers or the heterogeneous adhesion modes from MWCNTs or Glymo, the reinforcement effect was unequal along the whole fibre. Both strong bonding and relatively weak bonding coexist, leading to wider distribution of fragment lengths. It is interesting to note that the coexistence between strong interphase and weak interphase is similar to the biologic bone structure. Besides the apparent reinforcement effect from the strong interphase, the weak interphase serves to inhibit crack propagation or acts as mechanical damping elements [Gupta et al., 2005]. Consequently, the mid-homogeneous interphase with EPD fibre has the strongest interfacial strength which was confirmed by the shortest fragment length.

To provide an unique opportunity for the in-situ load and damage detection, we have exploited the self-diagnosing effects, as pilot approach, of semiconductive MWCNT-glass fibre in composites during tensile test. Fig. 9 shows the electrical resistance and stress as a function of applied strain. We identified basically three stages of resistance variation in i) linear, ii) non-linear, and iii) abrupt changes. At the first stage, the linear behaviour of the 

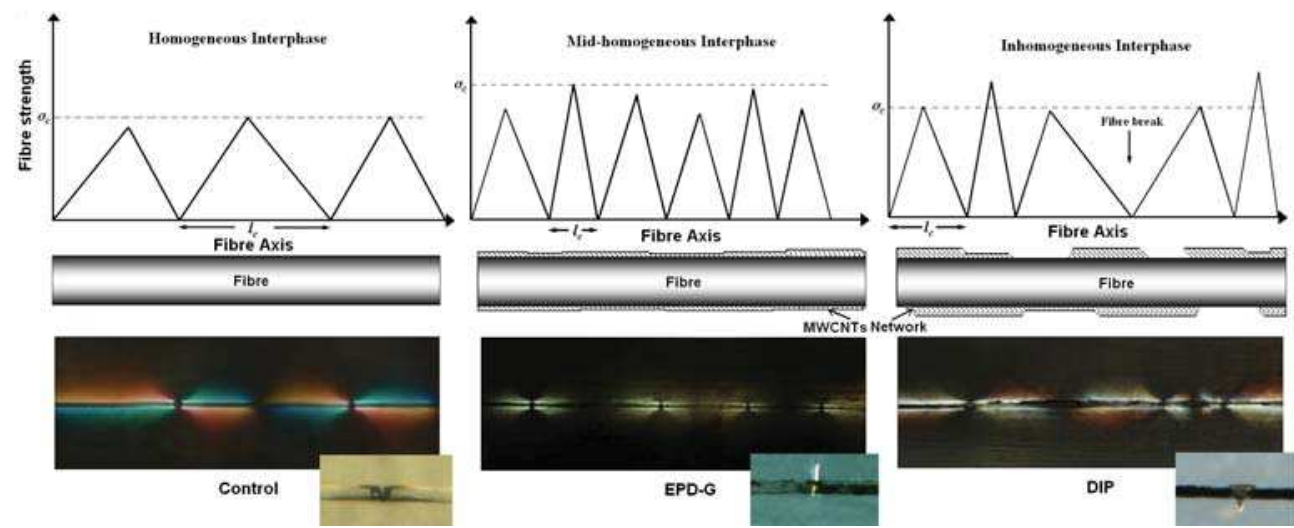

Fig. 8. Three kinds of stress profiles along the fibre axis as a function of position when fracture number reaches its saturation; the birefringence patterns are shown by crosspolarized light for saturation at a magnification of ten. Insert images are the enlarged views of broken points, the interfacial debonding failure mode for control and the matrix crack failure mode for EPD-G and DIP systems were observed.

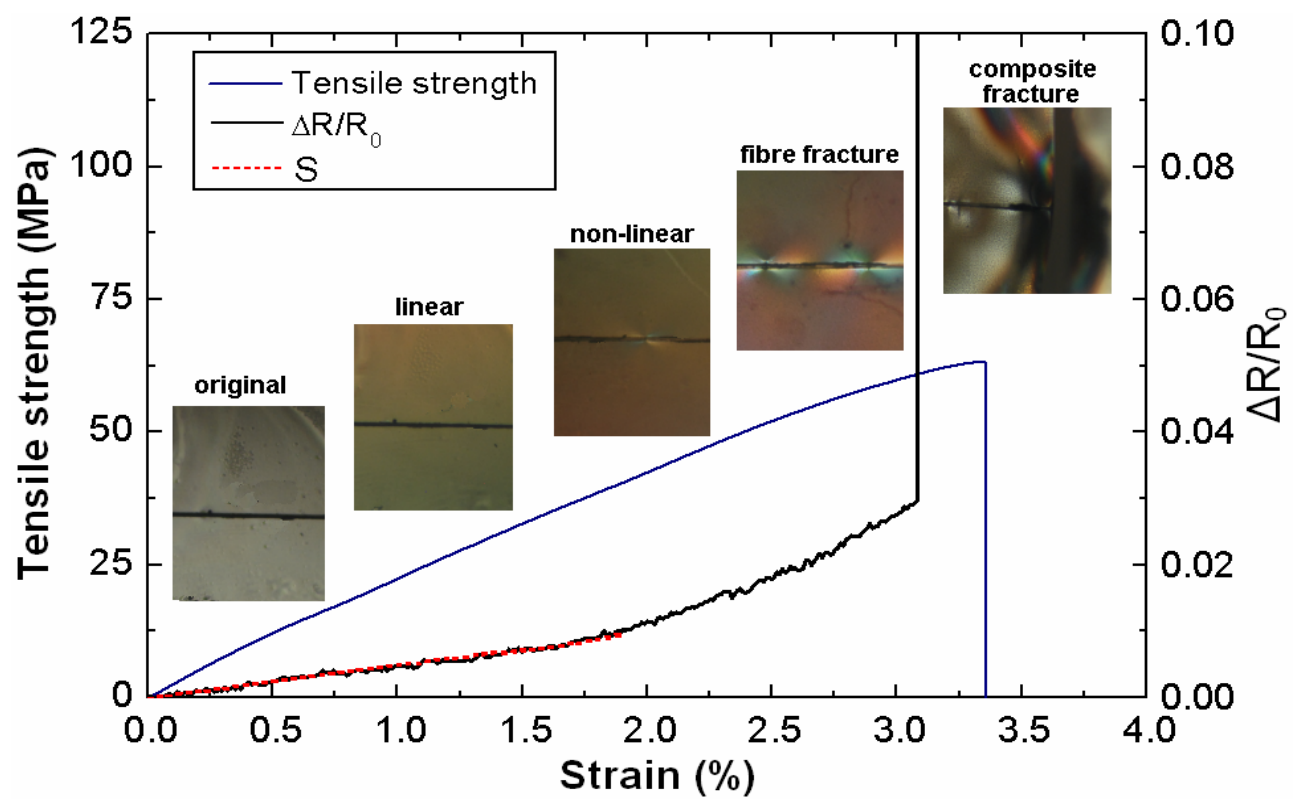

Fig. 9. Simultaneous change of electrical resistance and stress as a function of strain for single coated fibre/epoxy composite, the dashed $S$ is the straight line simulation of $\Delta R / R_{0}$ at the linear increasing stage. Inserted figures are the photoelastic profiles during tensile process corresponding to the $\Delta \mathrm{R} / \mathrm{R}_{0}$ value at the stages of original, linear, non-linear, fibre fracture and composite fracture. 
electrical resistance increased proportionally with strain up to approximately $1.5 \%$, which is possibly linked to the elastic deformation of the interphase. For strains higher than $1.5 \%$ the slope of the resistance-strain curve increased exponentially with strain. This exponential behaviour of resistance change is related to the interphase plastic deformation of CNT networks, associated with stress concentration before fibre breakage, increase of nanotubenanotube interspace and loss of junction points arising from permanent change in network shape during loading. This interphase deformation possibly caused irreversible resistance changes. At the third stage, the interphase failed completely and the resistant jumps "infinite" (The resistance exceeds measurable range). Finally, after interphase fracture, the coated fibre/epoxy composites failed at a strain of about $3.4 \%$. An important feature occurring in the measurements is that the three stages of the resistance variation are highly consistent and reproducible, thus making such single coated glass fibre as a small and sensitive rapid response mechanical sensor. Overall, our results show that the MWCNT coated fibre/epoxy composites possess a semiconductive interphase and the composite inherent damage can be monitored by measuring changes in electrical resistance in the early stage of damage. The piezoresistive effects of semiconductive MWCNTs-glass fibre and composites, therefore, provide a unique opportunity for an in-situ load and damage detection of the most widely used GFRPs, which, unlike other attempts, does not require additional sensors and dispersion of CNTs in polymer matrix.

We finally turn our attention to how the resistance of the interphase is sensitive to temperature and $\mathrm{T}_{\mathrm{g}}$ of polymer (Fig. 9). The resistance decreased monotonically with the increase of temperature, indicating a negative temperature coefficient (NTC) effect which reflects a typically semiconductive characteristic of the used MWCNTs. Most notably, we found a distinct transition on the $\Delta R / R_{o}$ curve from around $343 \sim 347 \mathrm{~K}$, which was almost coincident with the glass transition temperature, $\mathrm{T}_{\mathrm{g}} \approx 341 \sim 344 \mathrm{~K}$ shown in the curve measured by DSC. The transition of epoxy network at $\mathrm{T}_{\mathrm{g}}$ possibly induces break of some nanotube junction points and elongation of the interspaces between the CNTs, resulting in the variation of the resistance trends. Here, we can infer that the transition temperature detected through the semiconductive interphase is related to the brittle-ductile transition of epoxy in or near interphase when the temperature increases up to $\mathrm{T}_{\mathrm{g}}$. In turn, such transition at $T_{g}$ could induce variations of thermal residual stresses existing on the interface due to the coefficient of thermal expansion (CTE) mismatch between glass fibre and epoxy. The aforementioned nano-TA results suggest that the entangled nanotube network influences the local cross-linking density of epoxy in interphase resulting in a little different $\mathrm{T}_{\mathrm{g}}$ of interphase to that of bulk matrix.

\section{Conclusion}

A nanometer-scale hybrid coating layer based on styrene-butadiene copolymer with single or multi-walled carbon nanotubes (SWCNTs, MWCNTs) and/or nanoclays, as mechanical enhancement and environmental barrier layer, is applied to traditional glass fibres. The nanostructured and functionalised glass fibres show significantly improved both mechanical properties and environmental corrosion resistance. With low fraction of nanotubes in sizing, the tensile and bending strength of healed glass fibre increases remarkably. Besides, nanocomposite coatings result in enhanced fibre/matrix interfacial 
adhesion, indicating nanotube related interfacial toughening mechanisms. An increase of the local glass transition temperature in interphase regin, especially in distance less than 500 $\mathrm{nm}$ from the fibre surface was found, indicating higher crosslink density. The electrically insulating glass fibre deposited by MWCNTs leading to the formation of semiconductive MWCNT-glass fibres and in turn multifunctional fibre/polymer interphases. Our approach demonstrates for the first time that the techniques of conducting electrical resistance measurements could be applicable to glass fibres for in situ sensing of strain and damage; the techniques were previously limited to conductive and semiconductive materials. The electrical properties of the unidirectional fibre/epoxy composite show linear or nonlinear stress/strain and temperature dependencies, which are capable of detecting piezoresistive effects, early warning of fibre composite damage, as well as the local glass transition temperature. Based on our approach, the glass fibre - the most widely used reinforcement in composites globally-along with the surface electrical conductivity of MWCNTs will stimulate and realize a broad range of multifunctional applications.

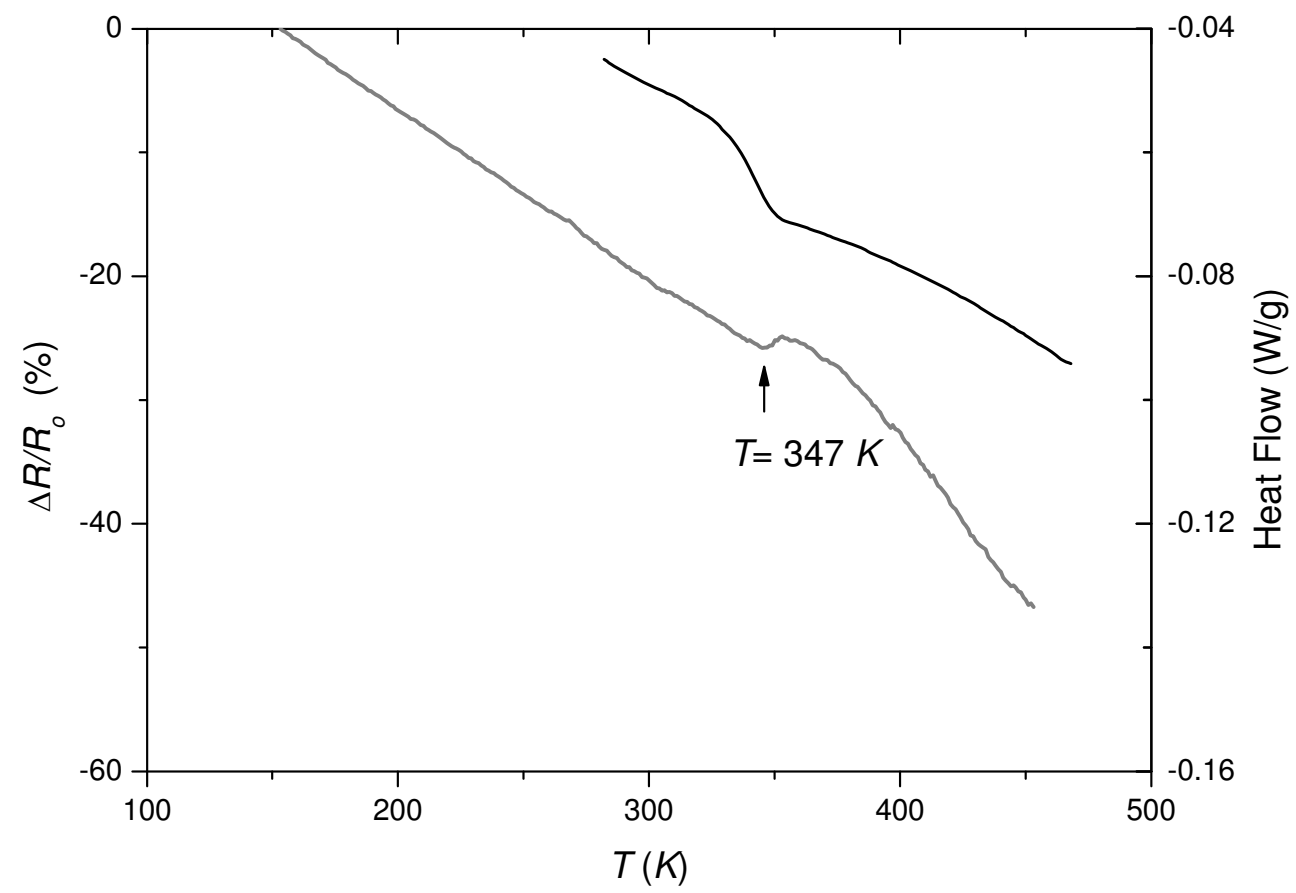

Fig. 10. Variability and trends of electrical resistance to temperature for MWCNTs-glass fibre/epoxy composites. The temperature dependence of $\Delta R / R_{o}$ and heat flow curve (black) obtained by the DSC. Note, the two different methods show similar transitions on the curves in the region of the glass transition temperature of epoxy.

\section{References}

Baughman, RH.; Zakhidov, AA. \& de Heer, WA. (2002). Carbon nanotubes--the route toward applications. Science, Vol., 297, 787-792. 
Bekyarova, E.; Thostenson, ET.; Yu, A.; Kim, H.; Gao, J.; Tang, J.; Hahn, HT.; Chou, TW.; Itkis, ME. \& Haddon, RC. (2007). Multiscale carbon nanoture-carbon fibre reinforcement for advanced epoxy composites. Langmuir, Vol., 23, 3970-3974.

Böger, L.; Wichmann, MHG.; Meyer, LO. \& Schulte, K. (2008). Load and health monitoring in glass fibre reinforced composites with an electrically conductive nanocomposite epoxy matrix. Composites Science and Technology, Vol., 68, 1886-1894.

Dzenis, Y. (2008). Structural nanocomposites. Science, Vol., 319, 419-420.

Gao, HJ.; Ji, BH.; Jäger, IL.; Arzt, D. \& Fratzl, P. (2003). Materials become insensitive to flaws at nanoscale: Lessons from nature. PNAS, Vol., 100, 5597-5600.

Gao, HJ. \& Ji, BH. (2003). Modeling fracture in nanomaterials via a virtual internal bond method. Engineering Fracture Mechanics, Vol., 70, 1777-1791.

Gao, SL.; Mäder, E. \& Plonka, R. (2007). Nanostructured coatings of glass fibres: Improvement of alkali resistance and mechanical properties. Acta Materialia, Vol., 55, 1043-1052.

Gao, SL.; Zhuang, RC.; Zhang, J.; Liu, JW. \& Mäder, E. (2010). Glass fibre with carbon nanotube networks as multifunctional sensor. Advanced Functional Materials, Vol., 20, 1885-1893.

Gupta, HS.; Wagermaier, W.; Zickler, GA.; Raz-Ben Aroush, D.; Funari, SS.; Roschger, P.; Wagner, HD. \& Fratzl, P. (2005). Nanoscale deformation mechanisms in bone. Nano Letter, Vol., 5, 2108-2111.

Gupta, S.; Zhang, QL.; Emarick, T.; Balazs, AC. \& Russell, TP. (2006). Entropy-driven segregation of nanoparticles to cracks in multilayered composite polymer structures. Nature Materials, Vol., 5, 229-233.

Thostenson, ET. \& Chou, TW. (2006). Carbon nanotube networks: sensing of distributed strain and damage for life prediction and self-healing. Advanced Materials, Vol., 18, 2837-2841.

Thostenson, ET. \& Chou, TW. (2008). Real-time in situ sensing of damage evolution in advanced fibre composites using carbon nanotube networks. Nanotechnology, Vol., 19, 215713.

Tombler, TW.; Zhou, CW.; Alexseyev, L.; Kong, J.; Dai, HJ.; Liu, L.; Jayanthi, CS.; Tang, M. \& Wu. SY. (2000). Reversible electromechanical characteristics of carbon nanotubes under local-probe manipulation. Nature , Vol., 405, 769-772.

Tyagi,S.; Lee, JY.; Buxton, GA. \& Balazs, AC. (2004). Using nanocomposite coatings to heal surface defects. Macromolecules, Vol., 37, 9160-9168.

Vigolo, B.; Penicaud, A.; Coulon, C.; Sauder, C.; Pailler, R.; Journet, C.; Bernier, P. \& Poulin, P. (2000). Macroscopic fibres and ribbons of oriented carbon nanotubes. Science, Vol., 290, 1331-1334.

Vigolo, B.; Poulin, P.; Lucas, M.; Launois, P. \& Bernier, P. (2002). Improved structure and properties of single-wall carbon nanotube spun fibres. Applied Physics Letter, Vol.,81, 1210-1212.

Veedu, VP.; Cao, AY.; Li, X.S.; Ma, KG.; Soldano, C. Kar, S. Ajayan, PM. Ghasemi-Nejhad. MN. (2006). Multifunctional composites using reinforced laminae with carbonnanotube forests. Nature Materials, Vol., 5, 457-462. 
Zhang, J.; Zhuang, RC.; Liu, JW.; Mäder, E.; Heinrich, G. \& Gao, SL. (2010). Functional interphases with multiwalled carbon nanotubes in glass fibre/epoxy composites. Carbon, Vol., 48, 2273-2281. 


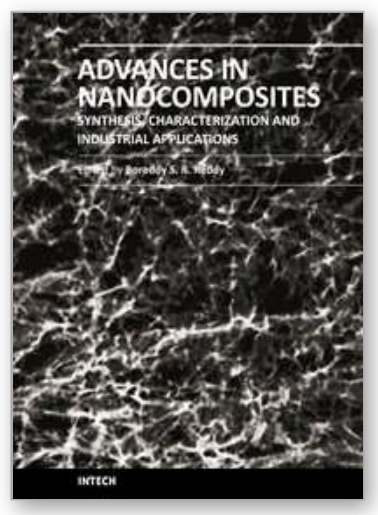

\author{
Advances in Nanocomposites - Synthesis, Characterization and \\ Industrial Applications
}

Edited by Dr. Boreddy Reddy

ISBN 978-953-307-165-7

Hard cover, 966 pages

Publisher InTech

Published online 19, April, 2011

Published in print edition April, 2011

Advances in Nanocomposites - Synthesis, Characterization and Industrial Applications was conceived as a comprehensive reference volume on various aspects of functional nanocomposites for engineering technologies. The term functional nanocomposites signifies a wide area of polymer/material science and engineering, involving the design, synthesis and study of nanocomposites of increasing structural sophistication and complexity useful for a wide range of chemical, physicochemical and biological/biomedical processes. "Emerging technologies" are also broadly understood to include new technological developments, beginning at the forefront of conventional industrial practices and extending into anticipated and speculative industries of the future. The scope of the present book on nanocomposites and applications extends far beyond emerging technologies. This book presents 40 chapters organized in four parts systematically providing a wealth of new ideas in design, synthesis and study of sophisticated nanocomposite structures.

\title{
How to reference
}

In order to correctly reference this scholarly work, feel free to copy and paste the following:

Shang-Lin Gao and Edith Mäder (2011). Nano Reinforcements in Surface Coatings and Composite Interphases, Advances in Nanocomposites - Synthesis, Characterization and Industrial Applications, Dr. Boreddy Reddy (Ed.), ISBN: 978-953-307-165-7, InTech, Available from:

http://www.intechopen.com/books/advances-in-nanocomposites-synthesis-characterization-and-industrialapplications/nano-reinforcements-in-surface-coatings-and-composite-interphases

\section{INTECH}

open science | open minds

\section{InTech Europe}

University Campus STeP Ri

Slavka Krautzeka 83/A

51000 Rijeka, Croatia

Phone: +385 (51) 770447

Fax: +385 (51) 686166

www.intechopen.com

\section{InTech China}

Unit 405, Office Block, Hotel Equatorial Shanghai

No.65, Yan An Road (West), Shanghai, 200040, China

中国上海市延安西路65号上海国际贵都大饭店办公楼405单元

Phone: +86-21-62489820

Fax: +86-21-62489821 
(C) 2011 The Author(s). Licensee IntechOpen. This chapter is distributed under the terms of the Creative Commons Attribution-NonCommercialShareAlike-3.0 License, which permits use, distribution and reproduction for non-commercial purposes, provided the original is properly cited and derivative works building on this content are distributed under the same license. 